\title{
Performance of a computer system for recording eye fixations using limbus reflection
}

\author{
DENNIS P. CARMODY, HAROLD L. KUNDEL, and CALVIN F. NODINE \\ Diagnostic Radiology Research Laboratory, Temple University School of Medicine \\ Philadelphia, Pennsylvania 19140
}

\begin{abstract}
A computer system was developed to measure and record locus and duration of the eye axis of gaze using a limbus reflection technique. System hardware and operating procedures for on-line data collection and correction are described. Algorithms are presented that separate fixations from movements. Based on these algorithms, system performance was measured in terms of spatial accuracy of eye fixations and temporal accuracy of saccadic movements.
\end{abstract}

Studies of visual search for small targets in both medical (Kundel \& Nodine, 1978; Kundel, Nodine, \& Carmody, 1978; Carmody, Note 1) and nonmedical images (Nodine, Carmody, \& Herman, 1979; Nodine, Carmody, \& Kundel, 1978) require inferences about the relation of the axis of the gaze to target detection and recognition. Consequently, a relatively accurate yet simple way is needed to study many observers, some of whom are highly trained professionals, such as radiologists, who must be instructed and tested within one session. For many years the Mackworth wide-angle and stand cameras (both from Polymetrics Company) were used to photograph the reflected image on the eye or the corneal reflection, respectively, on cine film. In either case, data reduction required a frame-by-frame analysis of the cine images, and even though a semiautomatic system was developed to analyze the films (Kundel \& Nodine, 1973), data analysis was laborious, and on-line operation was not possible. Since 1975, a limbus reflection technique has been used (NarcoBiosystems, Eye-Trac Model 200-1) interfaced to a PDP-11/40 computer (Digital Equipment Corporation). This paper describes the system and a study of the accuracy of the glasses during data acquisition.

\section{SYSTEM HARDWARE}

The operating system and a set of computer programs were developed to digitize data, separate blinks and movements from fixations, and, most importantly, correct the output of the glasses holding the eye movement sensors. The programs are written in FORTRAN ${ }^{1}$ for the RT-11 operating system of the PDP.11/40, but the logic of the approach is of general interest to

This research was supported in part by Grant GM21474, National Institute of General Medical Sciences, DHEW, and DAAG Grant 29-76-G-0313. Address reprint requests to Dennis P. Carmody, PhD, Diagnostic Radiology Research Laboratory, Temple University School of Medicine, Philadelphia, Pennsylvania 19140 scientists currently working with or contemplating development of an eye movement recording system.

The main design objective is to collect eye fixation locations and observer responses and to control the visual display in a simple, error-free way. This system consists of three random-access projectors (Kodak Ektagraphic), equipped with solenoid-driven shutters (Ilex Optical Company), that project $35-\mathrm{mm}$ slides onto a rear-projection screen. The projectors and shutters are operated under program control through digital interfaces. The observer's head is held in place by a tablemounted head and chin rest. A number of manual devices are used by the observer to signal decision events to the computer. For example, a numerical keypad is used to encode responses indicating rating scale judgments, and a sonic digitizing pen (Graf/Pen, Science Accessories Corporation) is used to identify target locations on the display. These peripheral devices are interfaced to the computer using digital input registers. Both a real-time clock and a bulk storage device, such as a tape or disk, are required to operate the system.

Eye position is sensed by photodiodes, mounted on the glass frames, when the eyes are illuminated by a pulsed infrared light. Horizontal eye movements are recorded from the right eye, and vertical movements are recorded from the lower left eyelid. The analog output from each eye is digitized by separate analogto-digital converters of the PDP-11/40 laboratory peripheral system module.

\section{STANDARD OPERATING PROCEDURES}

\section{General Procedure}

A calibration pattern is shown before and after each trial to adjust for the variations in the analog output of the glasses between trials due to changes in the position of the observer's head, cross talk between channels, and random variations. The display sequence thus consists of a calibration pattern, a clear field with a starting point indicated by a cross, the stimulus display, and finally, the calibration pattern again. In order to achieve an uninterrupted sequence of these displays, a threechannel projection system is used with the calibration slide on Channel 1, the preexposure cross on Channe1 2, and the stimulus 
picture on Channel 3. Electromechanical shutters are switched under computer control to change the display.

\section{Data Acquisition}

The calibration test pattern is a 5 by 5 array of $3 \mathrm{~mm}$ dots that subtend $.4 \mathrm{deg}$ at a $70-\mathrm{cm}$ viewing distance. The dots are $5 \mathrm{deg}$ apart, making a $20 \times 20 \mathrm{deg}$ display format. The four corner dots and the center dot are red; the rest are black. All 25 points are fixated during precalibration; only the five red dots are fixated during postcalibration. The precalibration data allow correction of fixations for the spatial nonlinearity of the system. The postcalibration data yield evidence of head movement during the test run.

Before each test picture, the observer fixates the 25 calibration points in order and pushes a button to indicate when each point is being steadily fixated. The fixation locus during calibration is digitized using $20 \mathrm{x}, \mathrm{y}$ sample pairs, and the mean values, $\overline{\mathbf{x}}$ and $\overline{\mathrm{y}}$, are stored and immediately displayed as a point on the operator's console. Sometimes inversion of calibration points or gross mismatches occur during the calibration. When these events occur, interrupting the trial during calibration and starting again results in better accuracy.

After the last calibration locus is entered, a predesignated starting point is shown for $2 \mathrm{sec}$, followed by the stimulus display. During the final $5 \mathrm{sec}$ of the fixation on the starting point, the digitization of eye locations is started at a rate of 800 samples/sec and is continued through the end of the display of the test pictures, which can last as long as $2 \mathrm{~min}$.

When the test is finished, the calibration pattern is redisplayed, and the four red corner points and red center point are entered by the observer as in the precalibration procedure.

\section{Data Correction}

The 25 precalibration data points are used to correct for nonlinearity of the output from the eye movement glasses. The five postcalibration data points are compared with the precalibration data points to determine how much movement occurred during the test run. By setting a pre-post difference threshold, runs can be accepted or rejected.

The square calibration pattern frequently results in an eye fixation pattern that is rhomboid or curvilinear. A typical example is shown in Figure $1 \mathrm{~A}$. These data are corrected by computing two polynomials of the form,

$$
\begin{aligned}
& X=A_{1}+A_{2} \bar{x}+A_{3} \bar{y}+A_{4} \overline{x y}+A_{5} \bar{x}^{2}+A_{6} \bar{y}^{2} \\
& Y=B_{1}+B_{2} \bar{x}+B_{3} \bar{y}+B_{4} \overline{x y}+B_{5} \bar{x}^{2}+B_{6} \bar{y}^{2},
\end{aligned}
$$

where $X$ and $Y$ are the corrected locations and $\bar{x}$ and $\bar{y}$ are the uncorrected measured locations (Wells, Note 2). The coefficients $A$ and $B$ are determined by using the Gauss elimination method (Kunz, 1957) to fit the 5 by 5 calibration pattern to a symmetrical 5 by 5 array. Figure 1B shows the data points after correction.

\section{SYSTEM PERFORMANCE}

System performance is described by the criteria of spatial accuracy of fixations and the temporal accuracy of saccades. Spatial accuracy is the displacement of the measured fixation location from the true location of the point being fixated on the display. This value depends on both the properties of the recording system and on the observer. Temporal accuracy indicates how closely the computed movement time approximates saccadic movements of known distances. Properties of the recording system that influence spatial and temporal accuracy include the digitizing sampling rate and the algorithms used to summarize the data.

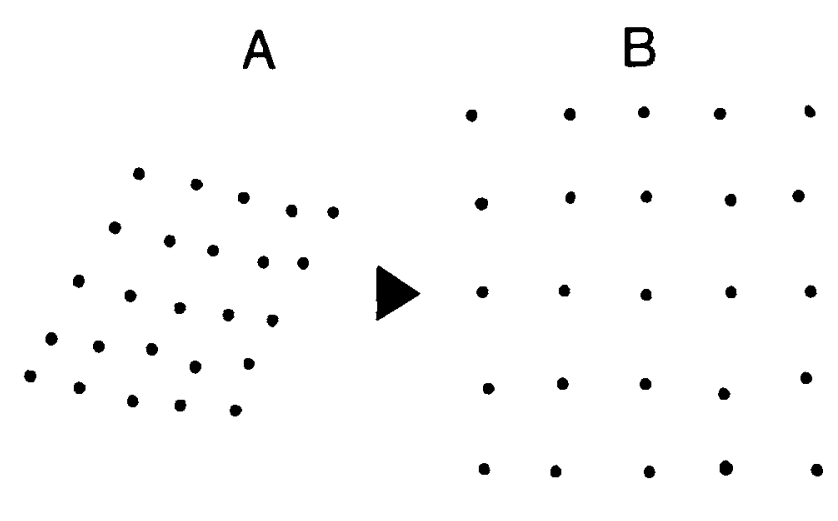

Figure 1. (A) A typical calibration pattern prior to correction. Through an algorithm, these data are corrected and scaled so that the values for $X$ and $Y$ are in centimeters on the viewscreen. (B) A corrected calibration pattem is also shown.

\section{Digitizing Eye Position}

Several factors influence the accuracy of measuring eye position: head movement, nonlinearity of sensor response, cross talk between the horizontal and vertical sensor channels, and fast noise in the analog output of the sensors. Head movement is measured by comparing pre-post differences in calibration data, and the spatial correction program corrects for nonlinearity and cross talk. A reduction in the error contribution of fast system noise is accomplished by preprocessing the analog signal before data storage.

Preprocessing consists of digitizing the analog signals at 800 samples/sec, grouped into 50 intervals of $20 \mathrm{msec}$. Within each interval, 16 analog samples are digitized and averaged to give an interval $\bar{x}$ and $\bar{y}$. This averaging smooths out the fast noise. Thus, every $20 \mathrm{msec}, \bar{x}$ and $\bar{y}$ are computed and stored in a buffer as uncorrected data. After data collection the spatial correction program is applied to these uncorrected data, and the results are permanently stored.

\section{Defining Fixations and Movements}

A nearest-neighbor rule defines the corrected interval data as fixations or movements according to a chosen radius. The distance from one interval to the next is calculated, and if it is less than the chosen radius, the intervals are combined into a fixation. A fixation locus $\left(X_{F}, Y_{F}\right)$ is calculated as the mean of the $X, Y$ values for the two intervals. The distance from this running mean to the next interval in the sequence is computed, and if it is less than the radius, a third interval is considered part of the fixation, and so on. This computation continues until an interval is encountered that falls outside the designated radius. The sum of all the interval durations in a fixation is the fixation duration.

When the interval $\bar{x}, \bar{y}$ exceeds the radius compared with both the previous and successive intervals, the interval is considered movement. Thus, fixations and move- 
ments are measured in groups of 20-msec intervals.

The choice of a critical radius infuences both the spatial accuracy of the corrected data and the number of intervals considered movement. A large radius will combine all data into one fixation, and a very small radius will define all data as movements. Therefore, a reasonable estimate of a critical radius is based on the accuracy of the corrected calibration data points with respect to the true position of the calibration points. From a sample of 105 eye movement records (Kundel et al., 1978), the average absolute accuracy of the 25 corrected calibration points is $.77 \mathrm{deg}$. Therefore a 1-deg radius is chosen as the critical radius for data reduction.

\section{Spatial Accuracy}

Spatial accuracy depends on machine and human parameters, the ideal limits being determined by the machine and the practical limits by the human. Since the glasses are attached to the head, any head movement shifts the axis of the gaze relative to the display, and unless head movement is measured, this can easily go unnoticed. Most recording systems have this problem and consequently employ bite boards, chinrests, headrests, and other devices to $15 y$ to immobiljze the head. When describing spatial accuracy, both machine accuracy, which is the performance limit of the apparatus with a totally rigid observer, and observer accuracy, which is achieved with a highly cooperative observer, are considered. The tendency to move the head is strong, and small movements are almost impossible to eliminate. Hence, there is a tendency for the axis of gaze to shift from the true position. These shifts are not separately measurable without instrumentation at present, and they confound the estimates of spatial accuracy.

Spatial accuracy is measured by having practiced observers, using the chin- and headrests, look at the 25 calibration points spaced at $5 \mathrm{deg}$ from a distance of $70 \mathrm{~cm}$. At this distance the entire display subtends a visual angle of $20 \mathrm{deg}$. After calibration, observers look at the .4-deg dots in the center and the four corner points for about 1 sec per point. Steady fixation for

Table 1

Spatial Accuracy (in Degrees) of Fixations Over Display Locations

\begin{tabular}{lcc} 
& \multicolumn{2}{c}{ Spatial Accuracy* } \\
\cline { 2 - 3 } Display Location & Horizontal & Vertical \\
\hline Upper Left & .25 & .42 \\
Upper Right & .25 & .50 \\
Center & .50 & .33 \\
Lower Left & .98 & .66 \\
Lower Right & .91 & .66 \\
Overall Mean & .58 & .51 \\
\hline
\end{tabular}

* Average displacement of measured fixation point from true point based on three observers looking at the .4-deg dots in the center and four display corners for about 1 sec each for five runs, yielding about 200 fixations.

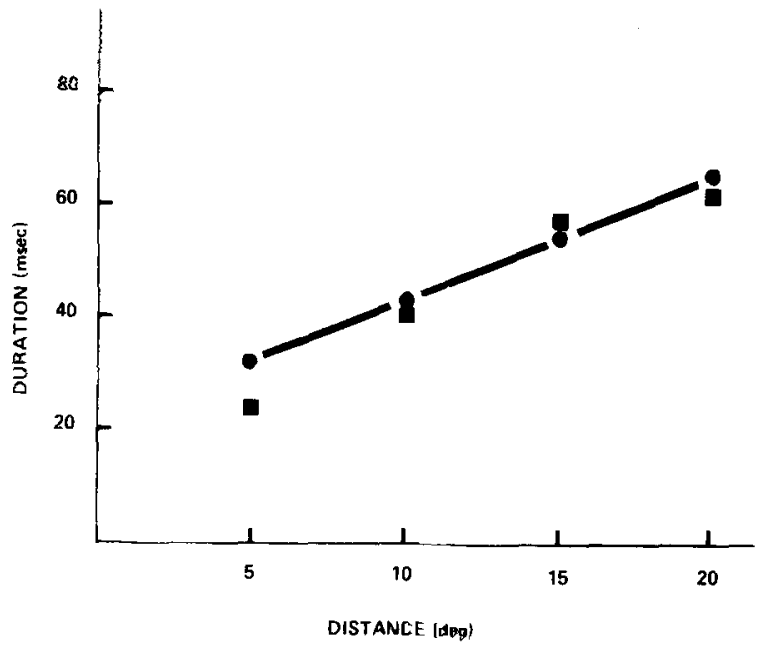

Figure 2. Saccadic duration as a function of distance. The squares ( $\bullet$ ) are the average data from the described digitized system and the circles $(\bullet)$ are from analog systems after Carpenter (1977, p. 57).

more than $1 \mathrm{sec}$ tends to produce noticeable drift that is frequently followed by a corrective jump back to a location near the original fixation point. Three observers were run five times; these data ase shown in Table 1. Using the correction algoritim for spatial location, the algebraic mean tends toward zero as the sample size increases. Therefore, accuracy is expressed as the mean absolute displacement of the measured location from the true location. These values include the error contribution of head shifts during the runs and are within the typical specifications published by the manufacturer of the glasses (Narco-Biosystems, Inc., Note 3).

\section{Temporal Accuracy}

Temporal accuracy of saccadic movements depends on the sampling rate. With the collection program, samples are averaged and stored 50 times/sec. Thus, saccadic movements are measured in intervals of $20 \mathrm{msec}$. Temporal accuracy is measured by having practiced observers look at points spaced at 5 -deg intervals. Three observers were run five times; the average data of movement durations for known distances from 5 to $20 \mathrm{deg}$ are shown in Figure 2. These data define movement time as a function of distance and agree with the data of Carpenter (1977, p. 57).

\section{CONCLUSIONS}

Much of our research deals with reading x-ray films and looking at pictures, and many of the observers are naive about eye movement recording and have little inclination to sit still for long periods of time while the experimenter adjusts the eye position recording glasses. Many different observers are used, which requires fre. quent changes in the settings of the sensors, and fre quently, we are forced to accept less than perfect adjustment. 
The system described here uses a chinrest, accepts nonlinear input, and on the average gives results that are spatially accurate within about $1 \mathrm{deg}$ of visual angle for fixations. We believe this is a good compromise between the high accuracy achievable by restraining the head, or measuring head motion and meticulously adjusting the sensors, and the ability to run a large number of observers quickly and easily.

\section{REFERENCE NOTES}

1. Carmody, D. P. Scanning strategies during global and segmented search for lung nodules. Manuscript in preparation.

2. Wells, E. S. On the correction of eyemovement data. Unpublished manuscript, 1978. (Available from the Diagnostic Radiology Research Laboratory, Temple University School of Medicine, Philadelphia, Pennsylvania 19140.)

3. Narco-Biosystems, Inc. Model 200 eye movement monitor operators manual, 1973.

\section{REFERENCES}

Carpenter, R. H. S. Movements of the eyes. London: Pion, 1977.
Kundel, H. L., \& Nodine, C. F. A computer system for processing eye-movement records. Behavior Research Methods \& Instrumentation, 1973, 5, 147-152.

Kundel, H. L., \& Nodine, C. F. Studies of eye movements and visual search in radiology. In J. W. Senders, D. F. Fisher, \& R. A. Monty (Eds.), Eye movements and the higher psychological functions. Hillsdale, N.J: Erlbaum, 1978.

Kundel, H. L., Nodine, C. F., \& Carmody, D. P. Visual scanning, pattern recognition, and decision-making in pulmonary nodule detection. Investigative Radiology, 1978, 13, 175-181.

Kunz, K. Numerical analysis. New York: McGraw-Hill, 1957.

Nodine, C. F., Carmody, D. P., \& Herman, E. Eye movements during visual search for artistically embedded targets. Bulletin of the Psychonomic Society, 1979, 13, 371-374.

Nodine, C. F., Carmody, D. P., \& Kundel, H. L. Searching for NINA. In J. W. Senders, D. F. Fisher, \& R. A. Monty (Eds.), Eye movements and the higher psychological functions. Hillsdale, N.J: Erlbaum, 1978.

\section{NOTE}

1. Copies of the FORTRAN program are available upon request from the authors.

(Received for publication August 20, 1979; revision accepted January $14,1980$. ) 